\title{
A simpler characterization of a spectral lower bound on the clique number
}

\author{
E. Alper Yıldırım
}

Received: 14 April 2009 / Accepted: 10 November 2009 / Published online: 24 November 2009

(C) Springer-Verlag 2009

\begin{abstract}
Given a simple, undirected graph $G$, Budinich (Discret Appl Math 127:535-543, 2003) proposed a lower bound on the clique number of $G$ by combining the quadratic programming formulation of the clique number due to Motzkin and Straus (Can J Math 17:533-540, 1965) with the spectral decomposition of the adjacency matrix of $G$. This lower bound improves the previously known spectral lower bounds on the clique number that rely on the Motzkin-Straus formulation. In this paper, we give a simpler, alternative characterization of this lower bound. For regular graphs, this simpler characterization allows us to obtain a simple, closed-form expression of this lower bound as a function of the positive eigenvalues of the adjacency matrix. Our computational results shed light on the quality of this lower bound in comparison with the other spectral lower bounds on the clique number.
\end{abstract}

Keywords Maximum clique $\cdot$ Maximum stable set $\cdot$ Stability number · Clique number $\cdot$ Graph spectra

Mathematics Subject Classification (2000) 05 C69 $\cdot$ 68R10 $\cdot 15$ A18

\section{Introduction}

Let $G=(V, E)$ be a simple, undirected graph with a vertex set $V=\{1,2, \ldots, n\}$ and an edge set $E$ consisting of $m$ edges. A clique $C \subseteq V$ is a set of mutually adjacent

This work is supported in part by TÜBİTAK (Turkish Scientific and Technological Research Council) through Grant 109M149. 
vertices. The clique number of $G$, denoted by $\omega(G)$, is the size of the maximum clique in $G$. A set $S \subseteq V$ is a stable set of $G$ if each pair of vertices in $S$ is mutually nonadjacent. The cardinality of the maximum stable set of $G$ is called the stability number of $G$ and is denoted by $\alpha(G)$. The maximum clique problem is equivalent to the maximum stable set problem on $\bar{G}$, the complement of $G$. We denote the $n \times n$ adjacency matrix of $G$ by $A_{G}$.

It is well-known that computing (or even approximating) the clique number of a graph is in general an NP-hard problem (Hastad 1999). The recent survey paper by Bomze et al. (1999) provides an account of the fairly rich literature including applications, formulations, exact algorithms, heuristics, bounds, and estimates. However, the clique number and a maximum clique can be computed in polynomial time for certain classes of graphs such as perfect graphs and complements of t-perfect graphs (Grötschel et al. 1988; Y1ldırım and Fan-Orzechowski 2006).

In the literature, several connections have been established between the clique or stability number of a graph and the spectral properties of the adjacency matrix or of the Laplacian matrix of $G$ (see e.g., Cvetković et al. 1979; Wilf 1986; Budinich 2003; Nikiforov 2006; Lu et al. 2007; Godsil and Newman 2008; Nikiforov 2009). In particular, Budinich (2003) proposed a lower bound on the clique number of a given graph $G$ by combining the quadratic programming formulation of the clique number due to Motzkin and Straus (1965) and the spectral decomposition of the adjacency matrix $A_{G}$. He established that this lower bound improves the previously known spectral lower bounds that rely on the Motzkin-Straus formulation. In this paper, we give a simpler, alternative characterization of this lower bound. In contrast with the characterization of the lower bound in Budinich (2003), our characterization requires the solution of a much simpler quadratic linesearch problem. For regular graphs, this simpler characterization allows us to obtain a simple, closed-form expression of this lower bound as a function of the positive eigenvalues of $A_{G}$. Our computational results shed light on the quality of the proposed lower bound in comparison with the other spectral lower bounds on the clique number.

This paper is organized as follows. In the remainder of this section, we define our notation. Section 2 discusses the continuous formulation of Motzkin and Straus (1965) and reviews several known lower bounds on the clique number. Section 3 presents the simpler, alternative characterization of the lower bound due to Budinich (2003). The closed-form expression of this lower bound for the class of regular graphs is the topic of Sect. 4. The computational results are presented in Sect. 5. Section 6 concludes the paper.

\subsection{Notation}

$\mathbb{R}^{n}$ and $\mathcal{S}^{n}$ denote the $n$-dimensional Euclidean space and the space of $n \times n$ real symmetric matrices, respectively. For $u \in \mathbb{R}^{n}, u_{i}$ denotes the $i$ th component of $u$. The complete graph on $n$ vertices is denoted by $K_{n}$. We reserve $e$ to denote the vector of all ones in the appropriate dimension. The $(n-1)$-dimensional unit simplex in $\mathbb{R}^{n}$ is denoted by $\Delta_{n}$, i.e., $\Delta_{n}:=\left\{x \in \mathbb{R}^{n}: e^{T} x=1, x \geq 0\right\}$. For a graph $G=(V, E)$ with $V=\{1, \ldots, n\}, A_{G} \in \mathcal{S}^{n}$ denotes the adjacency matrix of $G$. For a nonempty 
subset $C \subseteq V$, we use $\chi^{C}$ to denote the characteristic vector of $C$ scaled by $1 /|C|$ so that $\chi^{C} \in \Delta_{n}$.

\section{Formulation and lower bounds}

Given a simple, undirected graph $G=(V, E)$ with $|V|=n$, Motzkin and Straus (1965) established that

$$
1-\frac{1}{\omega(G)}=\max _{x \in \Delta_{n}} x^{T} A_{G} x
$$

which is a continuous formulation of a combinatorial optimization problem. In addition to paving the way for the use of continuous optimization methods to solve the maximum clique problem, this formulation plays a central role in the derivation of lower bounds on the clique number of $G$. By (1),

$$
\omega(G) \geq \mu(\tilde{x}):=\frac{1}{1-\tilde{x}^{T} A_{G} \tilde{x}}, \quad \text { for all } \tilde{x} \in \Delta_{n}
$$

Using the fact that $\tilde{x}=(1 / n) e \in \Delta_{n}$, it follows that

$$
\omega(G) \geq \frac{n^{2}}{n^{2}-2 m} \geq 1
$$

where $m=|E|$ is the number of edges of $G$. This bound matches the clique number for complete graphs $K_{n}$ and their complements.

In addition to computing the clique number, the formulation (1) can in some cases be used to identify a maximum clique. For any maximum clique $C \subseteq V, \chi^{C}$ is a global optimal solution of (1). However, a drawback of the Motzkin-Straus formulation (1) is the existence of global optimal solutions that do not correspond to characteristic vectors of maximum cliques of $G$. For instance, if $G$ is the 4-cycle $C_{4}$, then $\chi^{V}=(1 / 4) \mathrm{e}$ is a global optimal solution of (1) despite the fact that $V$ is not a clique of $G$.

In an attempt to circumvent this drawback of the Motzkin-Straus formulation, Bomze (1997) proposed the following regularized continuous formulation:

$$
1-\frac{1}{2 \omega(G)}=\max _{x \in \Delta_{n}}\left(x^{T} A_{G} x+\frac{1}{2} x^{T} x\right) .
$$

In contrast with (1), each local maximizer is strict and corresponds to the characteristic vector of a maximal clique (maximal with respect to inclusion). Also, each global maximizer is strict and corresponds to the characteristic vector of a maximum clique. Similarly to (1), this alternative formulation can be used to derive a lower bound on the clique number of $G$ :

$$
\omega(G) \geq \beta(\tilde{x}):=\frac{1}{2\left(1-\tilde{x}^{T} A_{G} \tilde{x}-(1 / 2) \tilde{x}^{T} \tilde{x}\right)}, \quad \text { for all } \tilde{x} \in \Delta_{n} .
$$


Despite the fact that the alternative formulation (4) has more appealing properties in terms of identifying cliques, the following lemma establishes that the Motzkin-Straus formulation actually yields better lower bounds.

Lemma 1 Let $G=(V, E)$ be a simple, undirected graph such that $|V|=n$. For each $\tilde{x} \in \Delta_{n}, \mu(\tilde{x}) \geq \beta(\tilde{x})$, i.e., the lower bound (2) obtained from the Motzkin-Straus formulation (1) is at least as good as the lower bound (5) obtained from the Bomze formulation (4).

Proof Let $\tilde{x} \in \Delta_{n}$. Note that $\tilde{x}^{T}\left(I+A_{G}\right) \tilde{x} \leq \tilde{x}^{T}\left(\mathrm{ee}^{T}\right) \tilde{x}=\left(\mathrm{e}^{T} \tilde{x}\right)^{2}=1$, where we used the fact that $\tilde{x} \geq 0$ and $I+A_{G}$ is a matrix consisting only of zeroes and ones. Therefore,

$$
\begin{aligned}
\frac{1}{\beta(\tilde{x})} & =2\left(1-\tilde{x}^{T} A_{G} \tilde{x}-\frac{1}{2} \tilde{x}^{T} \tilde{x}\right), \\
& =2-\tilde{x}^{T} A_{G} \tilde{x}-\tilde{x}^{T}\left(I+A_{G}\right) \tilde{x} \\
& \geq 1-\tilde{x}^{T} A_{G} \tilde{x}=\frac{1}{\mu(\tilde{x})}
\end{aligned}
$$

which completes the proof.

By Lemma 1, we henceforth restrict our attention to the Motzkin-Straus formulation (1). Most of the other lower bounds in the literature are obtained by combining the formulation (1) with the spectral theory of graphs. From this point on, we assume that $G=(V, E)$ is a connected graph without loss of generality, since the maximum clique problem can otherwise be decomposed into smaller problems on each connected component of $G$. We now collect some results about the spectra of such graphs. The reader is referred to Cvetković et al. (1979) for further details.

Theorem 1 Let $G=(V, E)$ be a connected graph with the adjacency matrix $A_{G} \in$ $\mathcal{S}^{n}$ such that $|V|=n \geq 2$ and let $\lambda_{1} \geq \lambda_{2} \geq \cdots \geq \lambda_{n}$ denote the spectrum of $A_{G}$.

1. $\sum_{i=1}^{n} \lambda_{i}=0$.

2. $1 \leq \lambda_{1} \leq n-1$ and $-\lambda_{1} \leq \lambda_{n} \leq-1$, i.e., $\lambda_{1}$ is the spectral radius of $A_{G}$.

3. $A_{G}$ is irreducible, which implies that there exists a positive eigenvector $u^{1} \in \mathbb{R}^{n}$, called the Perron eigenvector, corresponding to the simple eigenvalue $\lambda_{1}$, called the Perron root.

4. $e \in \mathbb{R}^{n}$ is an eigenvector of $A_{G}$ corresponding to $\lambda_{1}$ if and only if $G$ is a regular graph.

5. $A_{G}$ has exactly one positive eigenvalue $\lambda_{1}$ if and only if $G$ is a complete multipartite graph.

Given a graph $G$, let $\lambda_{1}>0$ and $u^{1} \in \mathbb{R}^{n}$ denote the Perron root and the positive Perron eigenvector of $A_{G}$, respectively. Using the feasible solution $\hat{x}:=\left(1 / s_{1}\right) u^{1} \in$ $\Delta_{n}$ of (1), where $s_{1}:=e^{T} u_{1}$, Wilf (1986) established that

$$
\omega(G) \geq \mu(\hat{x})=\frac{s_{1}^{2}}{s_{1}^{2}-\lambda_{1}}=\frac{\lambda_{1}}{s_{1}^{2}-\lambda_{1}}+1,
$$


with equality if $G$ is a complete graph. The lower bound (6) is an improvement over the lower bound (3).

More recently, Budinich (2003) proposed a new lower bound that makes use of all the eigenvectors of $A_{G}$. In particular, if $u^{1}, u^{2}, \ldots, u^{n}$ denote the eigenvectors of $A_{G}$ of unit Euclidean norm corresponding to the eigenvalues $\lambda_{1} \geq \lambda_{2} \geq \cdots \geq \lambda_{n}$, respectively, where $u^{1}>0$, one can construct a family of unit vectors

$$
y_{i}(\beta)=\beta u^{i}+\sqrt{1-\beta^{2}} u^{1} \in \mathbb{R}^{n}, \quad i=2,3, \ldots, n,
$$

where $\beta \in(-1,1)$. Then, $z_{i}(\beta):=\left(1 / e^{T} y_{i}(\beta)\right) y_{i}(\beta)$ is a feasible solution of (1) for $i=2,3, \ldots, n$ as long as $\beta \in\left[l_{i}, u_{i}\right]$, where

$$
\begin{aligned}
& l_{i}:=\max _{j: u_{j}^{i}>0} \frac{-u_{j}^{1}}{\sqrt{\left(u_{j}^{1}\right)^{2}+\left(u_{j}^{i}\right)^{2}}}<0, \quad i=2,3, \ldots, n, \\
& u_{i}:=\min _{j: u_{j}^{i}<0} \frac{u_{j}^{1}}{\sqrt{\left(u_{j}^{1}\right)^{2}+\left(u_{j}^{i}\right)^{2}}}>0, \quad i=2,3, \ldots, n .
\end{aligned}
$$

Let

$$
\begin{aligned}
g_{i} & :=\max _{\beta \in\left[l_{i}, u_{i}\right]} z_{i}(\beta)^{T} A_{G} z_{i}(\beta), \\
& =\max _{\beta \in\left[l_{i}, u_{i}\right]} \frac{\beta^{2} \lambda_{i}+\left(1-\beta^{2}\right) \lambda_{1}}{\left(\beta\left(e^{T} u^{i}\right)+\sqrt{1-\beta^{2}} s_{1}\right)^{2}}, \quad i=2,3, \ldots, n
\end{aligned}
$$

and let

$$
g^{*}:=\max _{i=2,3, \ldots, n} g_{i}
$$

It follows from (1) that

$$
\omega(G) \geq \frac{1}{1-g^{*}} .
$$

Unless $G$ is a complete multipartite graph, Budinich shows that (11) strictly improves upon (6).

A comparison of the three lower bounds reveals that (3) is the easiest to compute and is provably the weakest one. While (6) requires only the computation of the Perron root and the Perron eigenvector, one needs the full spectrum and the full set of eigenvectors to compute (11). 


\section{A simpler characterization}

Given a simple, connected, undirected graph $G=(V, E)$ with $|V|=n$, recall the Motzkin-Strauss formulation:

$$
1-\frac{1}{\omega(G)}=\max _{x \in \Delta_{n}} x^{T} A_{G} x .
$$

Let $u^{1}, u^{2}, \ldots, u^{n}$ denote the eigenvectors of $A_{G}$ of unit Euclidean norm corresponding to the eigenvalues $\lambda_{1} \geq \lambda_{2} \geq \cdots \geq \lambda_{n}$, respectively, where $u^{1}>0$. For $\tau \in \mathbb{R}$, let us define the following family of solutions:

$$
w_{i}(\tau):=\left(\frac{1}{s_{1}}\right) u^{1}+\tau\left(u^{i}-\left(\frac{s_{i}}{s_{1}}\right) u^{1}\right), \quad i=2,3, \ldots, n,
$$

where

$$
s_{i}:=\mathrm{e}^{T} u^{i}, \quad i=1,2, \ldots, n .
$$

It is easy to verify that $\mathrm{e}^{T} w_{i}(\tau)=1, i=2,3, \ldots, n$ for all $\tau \in \mathbb{R}$. Furthermore, since $u^{1}>0$, it follows that $w_{i}(\tau) \in \Delta_{n}$ if and only if $\tau \in\left[\tau_{l}^{i}, \tau_{u}^{i}\right]$, where

$$
\tau_{l}^{i}:=\max _{j: d_{j}^{i}>0} \frac{-u_{j}^{1}}{s_{1}\left(d_{j}^{i}\right)}<0, \quad \tau_{u}^{i}:=\min _{j: d_{j}^{i}<0} \frac{-u_{j}^{1}}{s_{1}\left(d_{j}^{i}\right)}>0, \quad i=2,3, \ldots, n,
$$

where

$$
d^{i}:=u^{i}-\left(\frac{s_{i}}{s_{1}}\right) u^{1}, \quad i=2,3, \ldots, n .
$$

Therefore, we can define the following linesearch problems:

$$
\begin{aligned}
v_{i}:= & \max _{\tau \in\left[\tau_{l}^{i}, \tau_{u}^{i}\right]} w_{i}(\tau)^{T} A_{G} w_{i}(\tau), \\
= & \max _{\tau \in\left[\tau_{l}^{i}, \tau_{u}^{i}\right]}\left(\lambda_{1}\left(\frac{1-s_{i} \tau}{s_{1}}\right)^{2}+\lambda_{i} \tau^{2}\right), \\
= & \max _{\tau \in\left[\tau_{l}^{i}, \tau_{u}^{i}\right]}\left\{\left(\frac{\lambda_{1}}{s_{1}^{2}}\right)-\left(\frac{2 \lambda_{1}\left(s_{i}\right)}{s_{1}^{2}}\right) \tau\right. \\
& \left.+\left(\lambda_{i}+\frac{\lambda_{1}\left(s_{i}\right)^{2}}{s_{1}^{2}}\right) \tau^{2}\right\}, \quad i=2,3, \ldots, n .
\end{aligned}
$$

Let us define

$$
v^{*}:=\max _{i=2,3, \ldots, n} v_{i}
$$


Since $w_{i}(\tau) \in \Delta_{n}$ for $\tau \in\left[\tau_{l}^{i}, \tau_{u}^{i}\right], i=2,3, \ldots, n$, it follows that

$$
\omega(G) \geq \frac{1}{1-v^{*}}
$$

The next proposition establishes that the lower bound (18) is exactly the same as the improved bound (11) due to Budinich.

Proposition 1 Let $G=(V, E)$ be a simple, undirected, connected graph. The lower bounds (18) and (11) agree.

Proof We prove the assertion by establishing a one-to-one correspondence between the feasible solutions of the linesearch problems (9) and (16). Let us fix $i^{*} \in\{2,3, \ldots, n\}$. Let $\beta^{*} \in\left[l_{i^{*}}, u_{i^{*}}\right]$, where $l_{i^{*}}$ and $u_{i^{*}}$ are given by (8). Therefore,

$$
\begin{aligned}
z_{i^{*}}\left(\beta^{*}\right) & =\frac{1}{e^{T} y_{i^{*}}\left(\beta^{*}\right)} y_{i}\left(\beta^{*}\right), \\
& =\frac{1}{\beta^{*}\left(s_{i^{*}}\right)+\sqrt{1-\left(\beta^{*}\right)^{2}} s_{1}}\left(\beta^{*} u^{i^{*}}+\sqrt{1-\left(\beta^{*}\right)^{2}} u^{1}\right),
\end{aligned}
$$

where $y_{i}\left(\beta^{*}\right)$ is given by (7), is a feasible solution of the linesearch problem (9). We will show that $z_{i}\left(\beta^{*}\right)$ corresponds to a feasible solution of the line search problem (16) for $i=i^{*}$.

Let us define

$$
\tau^{*}:=\frac{\beta^{*}}{\beta^{*}\left(s_{i^{*}}\right)+\sqrt{1-\left(\beta^{*}\right)^{2}} s_{1}} .
$$

Consider the linesearch problem (16) corresponding to $i=i^{*}$. By (12),

$$
\begin{aligned}
w_{i^{*}}\left(\tau^{*}\right) & =\tau^{*} u^{i^{*}}+\left(\frac{1-\tau^{*}\left(s_{i *}\right)}{s_{1}}\right) u^{1}, \\
& =\frac{\beta^{*}}{\beta^{*}\left(s_{i *}\right)+\sqrt{1-\left(\beta^{*}\right)^{2}} s_{1}} u^{i}+\frac{\sqrt{1-\left(\beta^{*}\right)^{2}}}{\beta^{*}\left(s_{i}\right)+\sqrt{1-\left(\beta^{*}\right)^{2}} s_{1}} u^{1}, \\
& =z_{i^{*}}\left(\beta^{*}\right),
\end{aligned}
$$

where we used (20) in the penultimate line and (19) in the last one. Since $z_{i}\left(\beta^{*}\right) \in \Delta_{n}$, it follows that $\tau^{*} \in\left[\tau_{l}^{i^{*}}, \tau_{u}^{i^{*}}\right]$, where $\tau_{l}^{i^{*}}$ and $\tau_{u}^{i^{*}}$ are given by (14). This implies that $v_{i} \geq g_{i *}$.

Conversely, let $\hat{\tau} \in\left[\tau_{l}^{i^{*}}, \tau_{u}^{i^{*}}\right]$. By (12) and (14),

$$
w_{i *}(\hat{\tau})=\hat{\tau} u^{i^{*}}+\left(\frac{1-\hat{\tau}\left(s_{i^{*}}\right)}{s_{1}}\right) u^{1}
$$


is a feasible solution of the linesearch problem (16) corresponding to $i=i^{*}$. Let us define

$$
\hat{\beta}:=\frac{\hat{\tau}}{\left((\hat{\tau})^{2}+\left(\frac{1-\hat{\tau}\left(s_{i} *\right)}{s_{1}}\right)^{2}\right)^{1 / 2}} .
$$

Then,

$$
\sqrt{1-(\hat{\beta})^{2}}=\left(1-\frac{(\hat{\tau})^{2}}{(\hat{\tau})^{2}+\left(\frac{1-\hat{\tau}\left(s_{i *}\right)}{s_{1}}\right)^{2}}\right)^{1 / 2}=\frac{1-\hat{\tau}\left(s_{i^{*}}\right)}{s_{1}\left((\hat{\tau})^{2}+\left(\frac{1-\hat{\tau}\left(s_{i *}\right)}{s_{1}}\right)^{2}\right)^{1 / 2}}
$$

where we used the fact that $1-\hat{\tau}\left(s_{i} *\right) \geq 0$ since otherwise $w_{i^{*}}(\hat{\tau})$ will necessarily have a negative component by (12). It follows that

$$
\begin{aligned}
z_{i}(\hat{\beta}) & =\frac{1}{\hat{\beta}\left(s_{i^{*}}\right)+\sqrt{1-(\hat{\beta})^{2}} s_{1}}\left(\hat{\beta} u^{i^{*}}+\sqrt{1-(\hat{\beta})^{2}} u^{1}\right) \\
& =\frac{1}{\hat{\tau}\left(s_{i^{*}}\right)+\left(\frac{1-\hat{\tau}\left(s_{i}\right)}{s_{1}}\right) s_{1}}\left(\hat{\tau} u^{i^{*}}+\left(\frac{1-\hat{\tau}\left(s_{i^{*}}\right)}{s_{1}}\right) u^{1}\right), \\
& =w_{i^{*}}(\hat{\tau}) .
\end{aligned}
$$

Since $w_{i^{*}}(\hat{\tau}) \in \Delta_{n}$, it follows that $\hat{\mu} \in\left[l_{i^{*}}, u_{i^{*}}\right]$, which implies that $g_{i^{*}} \geq v_{i^{*}}$.

Since $i^{*}$ is arbitrary, we have $g_{i}=v_{i}, i=2,3, \ldots, n$. Therefore, $g^{*}=v^{*}$, which implies that $1 /\left(1-v^{*}\right)=1 /\left(1-g^{*}\right)$.

Note that each linesearch problem (16) has a quadratic objective function and is therefore considerably simpler compared to its counterpart (9) required for the computation of (11). Furthermore, this alternative characterization leads to a simple, closed-form expression for regular graphs, which is the topic of the next section.

\section{Regular graphs}

A graph $G=(V, E)$ is said to be $k$-regular if each vertex in $V$ has exactly $k$ neighbors. In this section, we turn our attention into the special class of regular graphs. We first establish certain properties of the lower bounds on such graphs. Then, we present a closed-form expression of the lower bound (11) on this class of graphs using the simpler characterization outlined in Sect. 3.

First, we establish that the lower bounds (3) and (6) coincide on this class of graphs.

Lemma 2 Let $G=(V, E)$ be a connected, $k$-regular graph with $|V|=n$ vertices. Then, each of the lower bounds (3) and (6) is equal to $n /(n-k)$. 
Proof Since $2 m=n k$, the lower bound (3) is given by $n^{2} /\left(n^{2}-2 m\right)=n^{2} /\left(n^{2}-n k\right)=$ $n /(n-k)$. Let us consider the lower bound (6). Since $G$ is a regular graph of degree $k$, we have $A_{G} \mathrm{e}=k \mathrm{e}$, which implies that $k \in \mathbb{R}$ is the Perron root with the corresponding Perron eigenvector $u^{1}=(1 / \sqrt{n})$ e. Since $s_{1}=e^{T} u^{1}=\sqrt{n}$, it follows that the lower bound (6) is given by $s_{1}^{2} /\left(s_{1}^{2}-\lambda_{1}\right)=n /(n-k)$, which establishes the assertion.

Next, we establish that each of the three lower bounds (3), (6), and (11) (or, equivalently (18)) coincides with the clique number $\omega(G)$ on connected, regular, complete multipartite graphs.

Proposition 2 Let $G=(V, E)$ be a connected, $k$-regular, complete multipartite graph with $|V|=n$. Then, each of the three lower bounds (3), (6), and (11) [or, equivalently (18)] coincides with $\omega(G)$.

Proof Note that the vertices of $G$ can be partitioned into $t$ subsets such that each subset contains exactly $n / t$ mutually nonadjacent vertices. Since $G$ is a $k$-regular, complete multipartite graph, we have $k=(t-1)(n / t)$. Clearly, $\omega(G)=t$ since each subset can contribute at most one vertex to any clique and there exists a clique of size $t$. By Lemma 2, the lower bounds (3) and (6) are equal to $n /(n-k)=n /(n-(t-$ 1) $(n / t))=t=\omega(G)$. Since $G$ is a regular, connected, complete multipartite graph, it follows from Budinich (2003, Proposition 3) that the lower bounds (11) and (6) agree, which completes the proof.

\subsection{Closed-form expression of Budinich's lower bound}

In this section, we present a simpler, closed-form expression of the lower bound (11) for regular graphs relying on the alternative, simpler characterization of this lower bound given by (18).

Proposition 3 Let $G=(V, E)$ be a connected, $k$-regular graph with $|V|=n$. Let $\lambda_{1} \geq \lambda_{2} \geq \cdots \geq \lambda_{n}$ denote the eigenvalues of $A_{G}$ with the corresponding eigenvectors $u^{1}, u^{2}, \ldots, u^{n}$ of unit Euclidean norm, where $u^{1}>0$, and let $\mathcal{P}=\{i \in$ $\left.\{2,3, \ldots, n\}: \lambda_{i}>0\right\}$. Then, the lower bound (11) [or, equivalently (18)] is given by

$$
\omega(G) \geq \max \left\{\frac{n}{n-k}, \max _{i \in \mathcal{P}} \frac{1}{1-\frac{k}{n}-\left(\tau_{i}\right)^{2} \lambda_{i}}\right\},
$$

where

$$
\tau_{i}:=\max \left\{\min _{j: u_{j}^{i}<0} \frac{1}{n\left|u_{j}^{i}\right|}, \min _{j: u_{j}^{i}>0} \frac{1}{n u_{j}^{i}}\right\}, \quad i \in \mathcal{P} .
$$

Proof Note that $\mathcal{P}=\emptyset$ if and only if $G$ is a complete, regular multipartite graph by Theorem 1. In this case, the lower bound (18) is given by $n /(n-k)$ and coincides with the clique number $\omega(G)$ by Proposition 2 . 
Suppose that $G$ is not a complete multipartite graph. Since $G$ is a regular graph, it follows that $u^{1}=(1 / \sqrt{n}) e$ is the Perron eigenvector of $A_{G}$ corresponding to the Perron root $\lambda_{1}=k$ with $s_{1}=e^{T} u^{1}=\sqrt{n}$. Therefore, $s_{i}=e^{T} u^{i}=0, i=2,3, \ldots, n$, which implies that $d^{i}=u^{i}, i=2,3, \ldots, n$ by (15). By (14),

$$
\tau_{l}^{i}=\max _{j: u_{j}^{i}>0} \frac{-1}{n u_{j}^{i}}=-\min _{j: u_{j}^{i}>0} \frac{1}{n u_{j}^{i}}, \quad \tau_{u}^{i}=\min _{j: u_{j}^{i}<0} \frac{1}{n\left|u_{j}^{i}\right|}, \quad i=2,3, \ldots, n
$$

Therefore, we have, by (16), that

$$
v_{i}=\max _{\tau \in\left[\tau_{l}^{i}, \tau_{u}^{i}\right]}\left(\frac{k}{n}+\left(\tau^{2}\right) \lambda_{i}\right), \quad i=2,3, \ldots, n
$$

Clearly,

$$
\nu_{i}= \begin{cases}\frac{k}{n}+\left(\tau_{i}\right)^{2} \lambda_{i} & \text { if } i \in \mathcal{P} \\ \frac{k}{n} & \text { otherwise }\end{cases}
$$

where $\tau_{i}$ is defined as in (23). The assertion follows from (18) and (17).

We remark that Proposition 3 improves Wilf's lower bound for regular graphs given in Wilf (1986, Theorem 3).

Using the correspondence between the stability number of a graph and the clique number of its complement, we next establish a closed-form expression of the corresponding lower bound for the stability number of a regular graph. Let $G=(V, E)$ be a connected, $k$-regular graph and let $\lambda_{1} \geq \lambda_{2} \geq \cdots \geq \lambda_{n}$ denote the eigenvalues of $A_{G}$ with the corresponding eigenvectors $u^{1}, u^{2}, \ldots, u^{n}$ of unit Euclidean norm. It follows that $A_{G}=U \Lambda U^{T}$, where $U=\left[u^{1}, u^{2}, \ldots, u^{n}\right] \in \mathbb{R}^{n \times n}$ and $\Lambda=\operatorname{Diag}\left(\lambda_{1}, \lambda_{2}, \ldots, \lambda_{n}\right) \in \mathcal{S}^{n}$ is a diagonal matrix having $\lambda_{1}, \lambda_{2}, \ldots, \lambda_{n}$ as its diagonal entries. Therefore, the complement graph $\bar{G}$ is $(n-k-1)$-regular and its adjacency matrix $A_{\bar{G}}$ satisfies

$$
\begin{aligned}
A_{\bar{G}} & =\mathrm{ee}^{T}-I-A_{G}, \\
& =n u^{1}\left(u^{1}\right)^{T}-I-U \Lambda U^{T}, \\
& =U \operatorname{Diag}\left(n-k-1,-\lambda_{2}-1, \ldots,-\lambda_{n}-1\right) U^{T},
\end{aligned}
$$

where we used the facts that $u^{1}=(1 / \sqrt{n})$ e and $\lambda_{1}=k$. It follows that the eigenvalues of $\bar{G}$ are given by $\bar{\lambda}_{1}:=n-k-1 \geq \bar{\lambda}_{2}:=-\lambda_{n}-1 \geq \bar{\lambda}_{3}:=-\lambda_{n-1}-1 \geq \cdots \geq$ $\bar{\lambda}_{n}:=-\lambda_{2}-1$ with the corresponding eigenvectors $\bar{u}^{1}:=u^{1}$ and $\bar{u}^{i}:=u^{n-i+2}$ for $i=2,3, \ldots, n$. Using the fact that $\alpha(G)=\omega(\bar{G})$, we immediately obtain the following result.

Corollary 1 Let $G=(V, E)$ be a connected, $k$-regular graph with $|V|=n$. Let $\lambda_{1} \geq$ $\lambda_{2} \geq \cdots \geq \lambda_{n}$ denote the eigenvalues of $A_{G}$ with the corresponding eigenvectors 
$u^{1}, u^{2}, \ldots, u^{n}$ of unit Euclidean norm, where $u^{1}>0$. Let $\mathcal{Q}=\{i \in\{2,3, \ldots, n\}$ : $\left.\lambda_{i}<-1\right\}$. Then, the stability number of $G$ satisfies

$$
\alpha(G) \geq \max \left\{\frac{n}{k+1}, \max _{i \in \mathcal{Q}} \frac{1}{\frac{k+1}{n}+\left(\tau_{i}\right)^{2}\left(\lambda_{i}+1\right)}\right\},
$$

where

$$
\tau_{i}:=\max \left\{\min _{j: u_{j}^{i}<0} \frac{1}{n\left|u_{j}^{i}\right|}, \min _{j: u_{j}^{i}>0} \frac{1}{n u_{j}^{i}}\right\}, \quad i \in \mathcal{Q} .
$$

In a similar fashion, Corollary 1 improves Wilf's lower bound for regular graphs given in Wilf (1986, Theorem 4).

We close this section by relating Corollary 1 to a recent alternative characterization of the stability number of a regular graph due to Nikiforov (2009, Theorem 5). For a $k$-regular graph, Nikiforov established that

$$
\alpha(G)=\frac{1}{\frac{k+1}{n}+(\tau(u))^{2} \min _{u}\left\{u^{T} A u+1: e^{T} u=0,\|u\|=1\right\}},
$$

where

$$
\tau(u):=\max \left\{\min _{j: u_{j}<0} \frac{1}{n\left|u_{j}\right|}, \min _{j: u_{j}>0} \frac{1}{n u_{j}}\right\} .
$$

Since each $u^{i}, i \in \mathcal{Q}$ is a feasible solution of the minimization problem in the denominator, it follows that this characterization yields an alternative proof of Corollary 1 . While this characterization may be useful in terms of establishing lower bounds for the stability number of regular graphs, it does not offer any computational advantages since the nonconvex minimization problem in the denominator is still NP-hard in general.

\section{Computational results}

Recently, using an upper bound on the smallest eigenvalue $\lambda_{n}$ of $A_{G}$, Nikiforov (2009) established that

$$
\omega(G) \geq 1+\frac{2 m}{\left(n-\frac{2 m}{n}\right)\left(\frac{2 m}{n}-\lambda_{n}\right)},
$$

with equality if and only if $G$ is a complete regular multipartite graph.

In an attempt to assess and compare the quality of the four lower bounds (3), (6), (18) [or, equivalently (11)], and (26), we evaluated each bound on graphs from two different data sets. The first data set consists of the DIMACS collection of clique 
Table 1 Several statistics about the average approximation ratios

\begin{tabular}{lllll}
\hline & $(3)$ & $(6)$ & $(18)$ & $(26)$ \\
\hline Average & 0.1867 & 0.1901 & $\mathbf{0 . 2 7 8 0}$ & 0.2231 \\
Standard deviation & 0.1562 & 0.1555 & 0.2673 & 0.1609 \\
Maximum & 0.6696 & 0.6709 & $\mathbf{1 . 0 0 0 0}$ & 0.6782 \\
Minimum & 0.0127 & 0.0127 & $\mathbf{0 . 0 1 7 7}$ & 0.0174 \\
\hline
\end{tabular}

problems ${ }^{1}$ and the second set is composed of random graphs with different sizes and different edge densities.

We first evaluated each bound on each of the sixty six instances in the DIMACS collection of clique problems using MATLAB. The major work in computing each of the lower bounds (6), (18), and (26) was the computation of the eigenvalue decomposition of the adjacency matrix $A_{G}$.

As shown in Budinich (2003), the lower bound (18) [or, equivalently (11)] is an improvement over each of the lower bounds (3) and (6), which is also confirmed by our computational results. The lower bound (3), which is the easiest to compute, is always the weakest one among all four lower bounds. The lower bound (6) is tighter than (3). The lower bounds (18) and (26) always outperform (3) and (6). Our results reveal that the lower bounds (18) and (26) are generally incomparable. Among the four lower bounds, the lower bound (26) was the sharpest on thirty nine instances while Budinich's bound (18) outperformed the other bounds in the remaining twenty seven instances.

Rather than presenting the bounds for each instance, we report our computational results in terms of several statistics since they provide much more insight about the quality of each lower bound.

To this end, we computed, for each instance, the ratio of each lower bound to the corresponding clique number. This ratio can be viewed as an approximation factor. We restricted our analysis to the fifty five instances in the DIMACS collection whose clique numbers are known. For each lower bound (3), (6), (18), and (26), we report several statistics related to these approximation ratios in Table 1. Each boldface number denotes the largest one in its row.

Table 1 reveals that Budinich's lower bound (18) achieves the best average approximation ratio among all the lower bounds despite the fact that Nikiforov's lower bound (26) was the sharpest on the majority of the instances. Furthermore, while Budinich's lower bound agrees with the clique number on the DIMACS instances hamming6-2, hamming8-2, and hamming 10-2, the lower bound (26) is at most within about $68 \%$ of the clique number among all instances.

The distribution of the approximation ratios for each lower bound is depicted in Fig. 1. The horizontal axis represents the approximation ratios in ten equal intervals given by $(0,0.1],(0.1,0.2], \ldots,(0.9,10]$ and the vertical axis denotes the number of DIMACS instances whose approximation ratio falls into the corresponding interval.

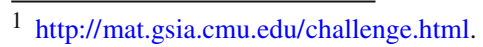




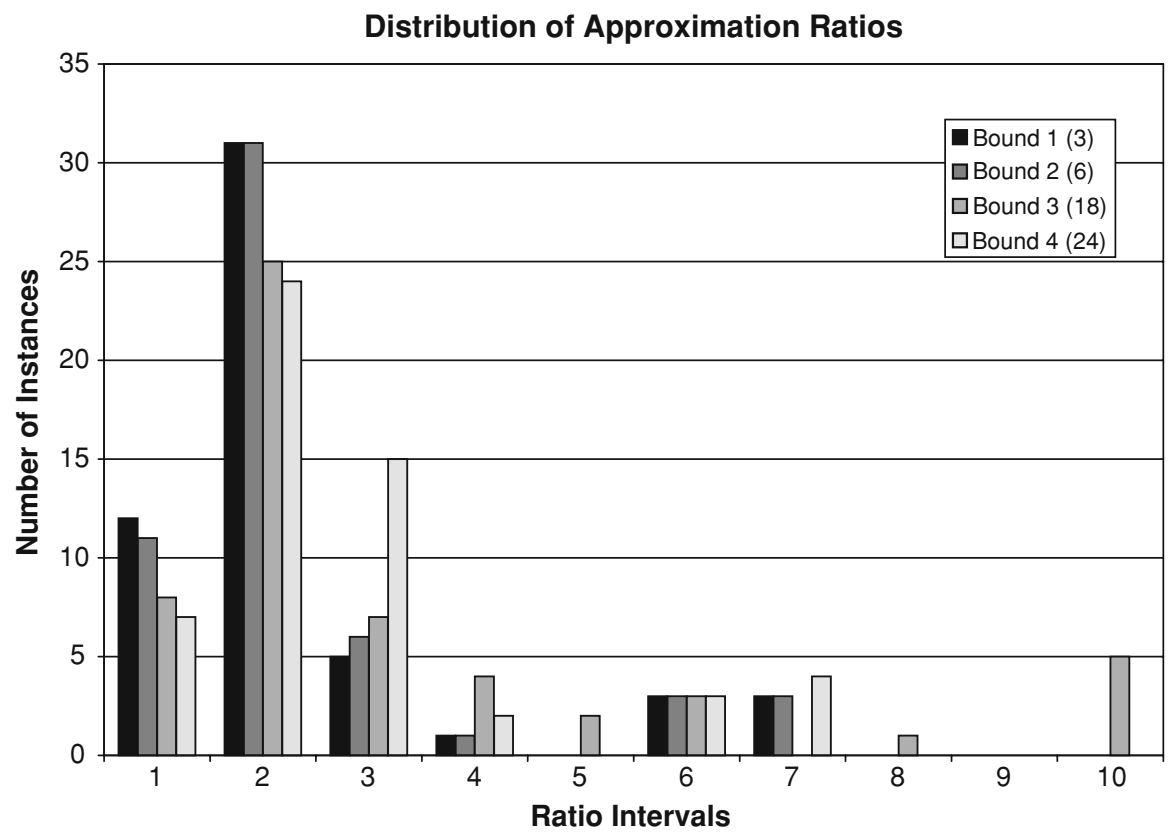

Fig. 1 Distribution of approximation ratios

Each of the four lower bounds is denoted by a different color as stated in the figure. A close examination of Fig. 1 reveals that Budinich's lower bound (18) achieves larger approximation ratios on more instances in comparison with the other lower bounds. We remark that the lower bound (18) is within $90 \%$ of the clique number on five instances whereas none of the other lower bounds achieves an approximation ratio of more than 0.7 on any of the instances.

In an attempt to assess the quality of the lower bounds on graphs with different edge densities, we generated random graphs with the number of vertices $n \in\{100,200\}$ and edge densities $\delta \in\{0.1,0.25,0.5,0.75,0.9\}$. For each choice of $n$ and $\delta$, we generated twenty random graphs. For each graph, we evaluated each of the four lower bounds and we computed the clique number using an integer programming solver. Similar to the DIMACS instances, we computed the approximation ratio for each bound. Table 2 presents the approximation ratios averaged over twenty instances for each choice of $n$ and $\delta$. The average clique number is denoted by $\bar{\omega}$.

Our computational results on random graphs reveal that the lower bound (3) is always the weakest, followed by (6). The lower bounds (18) and (26) outperform (3) and (6) on each instance. Similar to our results on DIMACS graphs, the bounds (18) and (26) are generally incomparable. An interesting observation is that Nikiforov's bound (26) outperforms Budinich's bound (18) on each instance with $\delta \in$ $\{0.1,0.25,0.5,0.75\}$. However, Budinich's bound is sharper than Nikiforov's bound on each instance with $\delta=0.9$. These results, which are summarized in Table 2, suggest that Budinich's bound is better on random graphs with high edge densities while 
Table 2 Computational results on random graphs

\begin{tabular}{llllll}
\hline$\delta$ & $\bar{\omega}$ & $(3)$ & $(6)$ & $(18)$ & $(26)$ \\
\hline$n=100$ & & & & & \\
0.1 & 4.00 & 0.2793 & 0.2859 & 0.2926 & $\mathbf{0 . 4 2 7 2}$ \\
0.25 & 5.65 & 0.2377 & 0.2431 & 0.2510 & $\mathbf{0 . 3 5 5 7}$ \\
0.5 & 9.30 & 0.2132 & 0.2176 & 0.2286 & $\mathbf{0 . 2 8 5 3}$ \\
0.75 & 17.05 & 0.2291 & 0.2336 & 0.2515 & $\mathbf{0 . 2 6 3 1}$ \\
0.9 & 30.80 & 0.2967 & 0.3023 & $\mathbf{0 . 3 3 4 8}$ & 0.3084 \\
$n=200$ & & & & & \\
0.1 & 4.15 & 0.2690 & 0.2721 & 0.2755 & $\mathbf{0 . 4 3 1 2}$ \\
0.25 & 6.60 & 0.2039 & 0.2061 & 0.2098 & $\mathbf{0 . 3 1 6 8}$ \\
0.5 & 11.00 & 0.1806 & 0.1825 & 0.1879 & $\mathbf{0 . 2 4 8 9}$ \\
0.75 & 21.25 & 0.1853 & 0.1873 & 0.1959 & $\mathbf{0 . 2 1 7 9}$ \\
0.9 & 40.85 & 0.2313 & 0.2336 & $\mathbf{0 . 2 4 9 6}$ & 0.2444 \\
\hline
\end{tabular}

Nikiforov's bound is sharper on sparser random graphs. We remark that Nikiforov's bound achieves very good approximation ratios for $\delta=0.1$.

For fixed $n$, Table 2 indicates that the approximation ratios do not seem to follow a well-defined pattern with respect to the edge density of graphs for any bound. For fixed $\delta$, the approximation ratios usually deteriorate as the number of vertices $n$ increases, with the sole exception of Nikiforov's bound (26) for $\delta=0.1$.

For each fixed value of $n$ and $\delta$, the approximation ratios were very close to one another as indicated by standard deviations around 0.01 . Therefore, reporting average approximation ratios seems meaningful. We remark that the computational results reported in Budinich (2003) are generally consistent with our results on random graphs.

In our computational experiments, we used each of the two characterizations (11) and (18) to compute Budinich's lower bound. While both bounds agree in theory, our computational results revealed that the original characterization (11) always yields slightly smaller values in comparison with the simpler characterization (18) presented in this paper. This might be due to the fact that the original characterization is more prone to numerical errors. It follows that the simpler characterization seems to be more appealing also from a computational point of view.

\section{Concluding remarks}

In this paper, we presented a simpler, alternative characterization of a spectral lower bound on the clique number due to Budinich (2003). Our characterization leads to a closed-form expression of this lower bound on regular graphs. Our computational results shed light on the quality of this lower bound in comparison with other spectral lower bounds on the clique number.

Given the hardness of any nontrivial approximation of the clique number, the construction of efficiently computable lower bounds may have significant implications 
towards the computation of clique number on larger graphs. For instance, good lower bounds can lead to a considerable reduction in the running time of search algorithms such as branch-and-bound. In the near future, we intend to continue our work on obtaining efficient upper and lower bounds by considering various tractable inner and outer approximations to the continuous formulation (1) of the clique number.

Acknowledgments We gratefully acknowledge the insightful comments and suggestions of two anonymous referees.

\section{References}

Bomze IM (1997) Evolution towards the maximum clique. J Glob Optim 10:143-164

Bomze IM, Budinich M, Pardalos PM, Pelillo M (1999) The maximum clique problem. In: Du DZ, Pardalos PM (eds) Handbook of combinatorial optimization (supplement volume A). Kluwer, Boston, pp $1-74$

Budinich M (2003) Exact bounds on the order of the maximum clique of a graph. Discret Appl Math 127:535-543

Cvetković DM, Doob M, Sachs H (1979) Spectra of graphs. Pure and applied mathematics. Academic Press, New York

Godsil CD, Newman MW (2008) Eigenvalue bounds for independent sets. J Comb Theory Ser B 98:721734

Grötschel M, Lovász L, Schrijver A (1988) Geometric algorithms and combinatorial optimization. Springer, New York

Hastad J (1999) Clique is hard to approximate within $n^{1-\epsilon}$. Acta Math 182(1):105-142

Lu M, Liu H, Tian F (2007) Laplacian spectral bounds for clique and independence numbers of graphs. J Comb Theory Ser B 97:726-732

Motzkin TS, Straus EG (1965) Maxima for graphs and a new proof of a theorem of Turán. Can J Math 17:533-540

Nikiforov V (2006) The smallest eigenvalue of $K_{r}$-free graphs. Discret Math 306:612-616

Nikiforov V (2009) More spectral bounds on the clique and independence numbers. J Comb Theory Ser B. doi:10.1016/j.jctb.2009.01.003

Wilf HS (1986) Spectral bounds for the clique and independence numbers of graphs. J Comb Theory Ser B 40:113-117

Yıldırım EA, Fan-Orzechowski X (2006) On extracting maximum stable sets in perfect graphs using Lovász's theta function. Comput Optim Appl 33(2-3):229-247 